\title{
Synthesis and characterization of carbon aerogels with acidizing process
}

\author{
Shasha Wang ${ }^{1,2, a}$, Meifang Yan ${ }^{1,2, b}$, Haihua $\mathrm{Li}^{1,2, \mathrm{c}}$ Lihui Zhang ${ }^{1,2, \mathrm{~d}}$, \\ and Zhenfa Liu ${ }^{1,2, \mathrm{e}^{*}}$
}

1. Institute of Energy Resources, Hebei Academy of Science, Shijiazhuang Hebei 050081, China

2. Hebei Engineering Research Center for Water Saving in Industry, Shijiazhuang Hebei 050081 , China

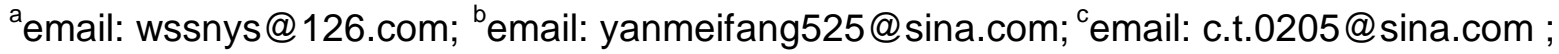

demail: zlhkxy@126.com; eemail: liuzhenfa@hebut.edu.cn

Keywords: Carbon aerogel, Acidizing process, Specific surface area, SEM

Abstract. Phloroglucinol-resorcinol-formaldehyde (PRF) carbon aerogels were synthesized using $\mathrm{Na}_{2} \mathrm{CO}_{3}$ as the catalyst. Acidizing process was introduced to the sol-gel reactions system. The results show that the acidizing process of trifluoroacetic acid acetone solution can decrease the shrinkage factor of carbon aerogel, increase absorption capacity and specific surface area. The SEM images of carbon aerogel with acidizing process possess closer network structure and excellent connectivity, the holes between the network are abundant. The XRD patterns show the acidizing process of trifluoroacetic acid acetone solution can enhance the graphitization degree of carbon aerogels.

\section{Introduction}

Carbon aerogels are novel porous carbon materials that have received considerable attention in the literature over the past decade or so[1,2]. These materials are very porous with interesting properties such as: low density, high surface area, continuous open porosity and electrical conductivity, can be obtained by sol-gel polycondensation of certain organic monomers such as resorcinol with formaldehyde. However, structure collapse of the obtained wet organic aerogels by sol-gel method was occurred due to the presence of the surface tension of solvent, which greatly affect the properties of carbon aerogels. Usually, this problem was solved by supercritical drying, but the high cost of this technology, complex process, and a certain dangerous nature, hinder the practical application of carbon aerogels. Therefore, researchers are exploring atmospheric drying instead of supercritical drying in order to optimize the preparation process [3].

In this paper, acidizing process was introduced to the sol-gel reactions system to enhance cross link intensity. Phloroglucinol-resorcinol-formaldehyde (PRF) caborn aerogels were synthesized using $\mathrm{Na}_{2} \mathrm{CO}_{3}$ as the catalyst, by aging, acidification, solvent exchange, atmospheric drying, high temperature carbonization process.

\section{Experimental}

\section{Synthesis}

Carbon aerogel samples were prepared by the sol-gel polymerization method. Resorcinol (R), Phloroglucinol $(\mathrm{P})$, and formaldehyde $(\mathrm{F})$ were dissolved in deionized water $(\mathrm{W})$ and sodium carbonate as a catalyst (C). The molar ratios of reagents was set as $\mathrm{P} / \mathrm{R}=0.15 ;(\mathrm{P}+\mathrm{R}) / \mathrm{F}=0.5 ;(\mathrm{P}+\mathrm{R}) / \mathrm{C}=800$, and $\mathrm{R} / \mathrm{W}=0.06$. The monomer concentration of reactants $(\mathrm{P}+\mathrm{R}+\mathrm{F})$ in solution was $45 \%$. To ensure a homogeneous mixture, the reagents were shocked by ultrasonic oscillator in sealed glass moulds until gelification. This gel was heated in test tubes at $50{ }^{\circ} \mathrm{C}$ for $72 \mathrm{~h}$ and then $80{ }^{\circ} \mathrm{C}$ for $24 \mathrm{~h}$ and the PRF hydrogels were obtained. The obtained PRF hydrogels were treated by acidizing process, in this process the PRF hydrogels were submerged in 3\% (volume fraction) trifluoroacetic acid solution for $72 \mathrm{~h}$, and then aged PRF hydrogels with cross-linked netlike structure were obtained. Solvent 
exchange for acetone was carried out by changing solution after every $24 \mathrm{~h}$ for 3 times. Drying was carried out at ambient pressure. The dried PRF aerogels were carbonized at $900{ }^{\circ} \mathrm{C}$ for $3 \mathrm{~h}$ under a flowing high purity nitrogen atmosphere $(100 \mathrm{~mL} / \mathrm{min})$.

Three carbon aerogel samples were generated: CA without acidizing process; PRF-acidizing-carb(1) with acidizing process by $3 \%$ (volume fraction) trifluoroacetic acid aqueous solution; PRF-acidizing-carb(2) with acidizing process by 3\% (volume fraction) trifluoroacetic acid acetone solution.

\section{Characterizations}

The surface morphologies of carbon aerogels were observed by S-4800-I (Hitachi, Japan) scanning electron microscopy (SEM). Nitrogen adsorption and desorption were measured using ASAP 2420 surface area analyzer (Micromeritics, America). The samples were degassed at $350{ }^{\circ} \mathrm{C}$ overnight prior to the adsorption measurements. The specific surface areas were determined by BET method. And pore size distributions were calculated by the BJH method from adsorption branch of the isotherms. X-ray diffraction (XRD) patterns of carbon aerogels were obtained by UItima IV X-ray diffractometer (Rigaku, Japan).

\section{Results and discussion}

\section{Condition of shrinkage}

The samples obtained are regular cylindrical solids. The condition of shrinkage can be tested by diameter comparison of samples before and after carbonization. As shown in Table 1, the shrinkage condition of PRF-acidizing-carb(2) was improved greatly with acidizing process. This result indicate acidizing process of trifluoroacetic acid acetone solution (3\%) improve the degree of crosslinking obviously, is advantageous to the formation of more stable network polymer and avoid structure collapse during carbonization.

Table 1 Diameter comparison of samples before and after carbonization

\begin{tabular}{cccc}
\hline Samples & $\begin{array}{c}\text { Diameier before } \\
\text { carbonization/cm }\end{array}$ & $\begin{array}{c}\text { Diameier after } \\
\text { carbonization/cm }\end{array}$ & $\begin{array}{c}\text { Shrinkage } \\
\text { factor/\% }\end{array}$ \\
\hline PRF-acidizing-carb(1) & 2.200 & 1.759 & 20.04 \\
PRF-acidizing-carb(2) & 2.137 & 1.903 & 15.63 \\
CA & 2.310 & 1.424 & 38.35 \\
\hline
\end{tabular}

\section{Physical characteristics}

The pore structure of the carbon aerogels were investigated by low temperature nitrogen adsorption measurements. The $\mathrm{N}_{2}$ adsorption/desorption isotherms and the pore size distribution (PSD) curves are shown in Fig. 1 and Fig. 2, respectively. The porosity data deduced from the isotherms are listed in Table 2.

These isotherms were the type IV indicating monolayer adsorption of possessing a capillary condensation phenomenon happening on the surface of carbon aerogels [4]. The hysteresis loops at relative pressures above 0.6 clearly show the presence of mesoporosity[5]. The nitrogen adsorption capacity of PRF-acidizing-carb(2) is the highest, indicating the specific surface area of PRF-acidizing-carb(2) is the largest $\left(697.65 \mathrm{~m}^{2} \cdot \mathrm{g}^{-1}\right)$. 


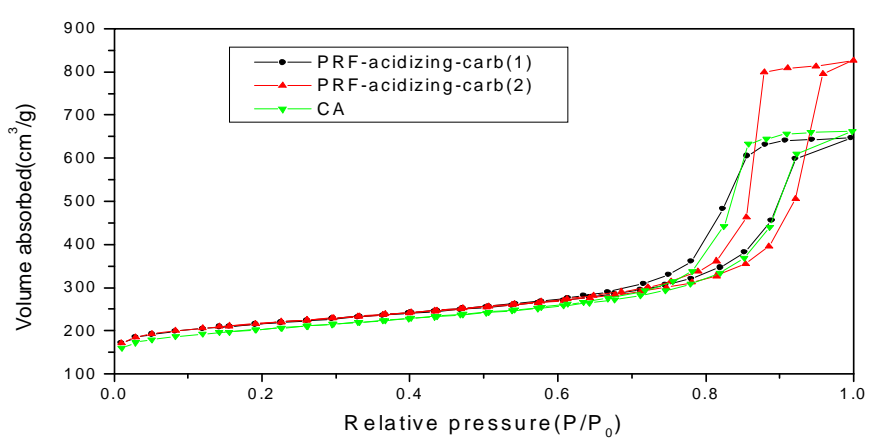

Fig. 1 - Low temperature nitrogen isotherms of carbon aerogels. $\bullet$ PRF-acidizing-carb(1), $\Delta$ PRF-acidizing-carb(2), $\mathbf{V}$ CA.

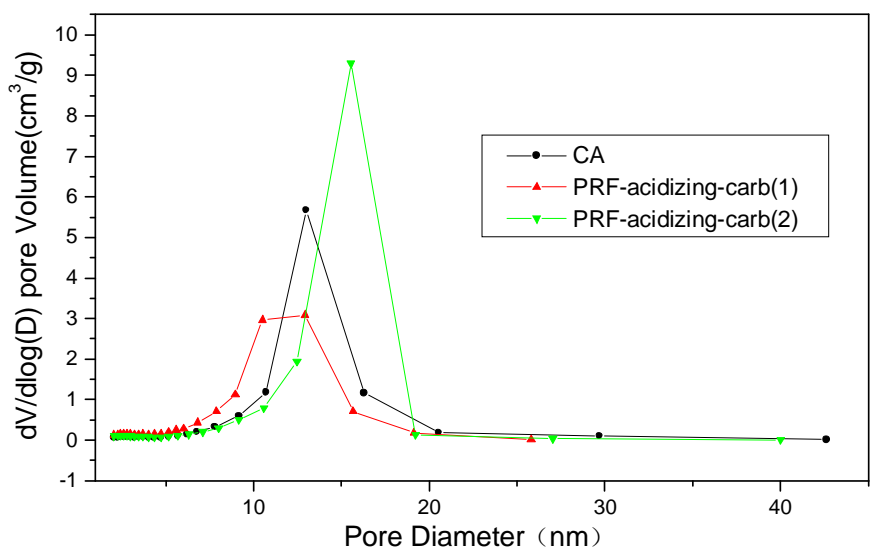

Fig. 2 - Mesopore size distribution from BJH method. •CA, $\Delta$ PRF-acidizing-carb(1), $\boldsymbol{\nabla}$ PRF-acidizing-carb(2).

The mesopore size distribution of three samples mainly focus on $10 \mathrm{~nm}-20 \mathrm{~nm}$, PRF-acidizing-carb(2) display a narrow distribution at $15 \mathrm{~nm}$. After acidizing process, the surface area of PRF-acidizing-carb(1) and PRF-acidizing-carb(2) are larger than CA, indicate acidizing process has positive effects on the formation of abundant network structures.

Table 2 Data reduced from low temperature nitrogen adsorption.

\begin{tabular}{cccc}
\hline Samples & $\mathrm{S}_{\mathrm{BET}}\left(\mathrm{m}^{2} \cdot \mathrm{g}^{-1}\right)$ & $V_{\text {tot }}\left(\mathrm{cm}^{3} \cdot \mathrm{g}^{-1}\right)$ & $\mathrm{R}(\mathrm{nm})$ \\
\hline PRF-acidizing-carb(1) & 664.03 & 1.00 & 5.77 \\
PRF-acidizing-carb(2) & 697.65 & 1.28 & 7.33 \\
$\mathrm{CA}$ & 606.22 & 1.03 & 6.24 \\
\hline $\mathrm{S}_{\mathrm{BET}}$, specific surface area from BET & model; $V_{\text {tot }}$, pore volume at \\
$\mathrm{p} / \mathrm{p} 0 \rightarrow 1 ; \mathrm{R}$, average pore size.
\end{tabular}

\section{Structural characterization}

\section{SEM morphologies of carbon aerogels}

SEM images of the surface morphology of three samples are depicted in Fig.1 (a)-(c). The clusters and holes of the sample without acidizing process are variant, made of slender network structure. The sample CA is typical disorder porous material. Compared with the sample CA, PRF-acidizing-carb(1) and PRF-acidizing-carb(2) possess closer network structure, the holes between the network are abundant, have excellent connectivity. 


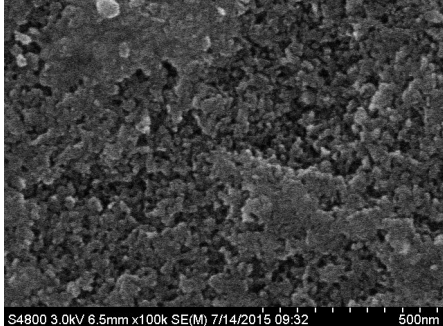

(a)

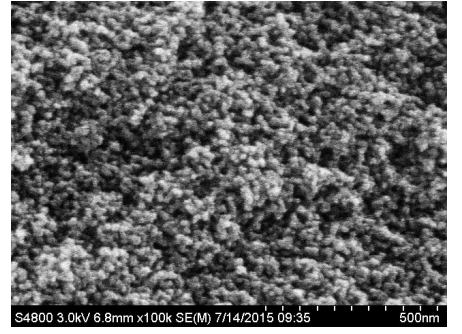

(b)

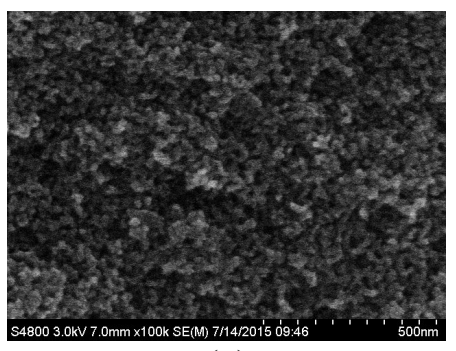

(c)

Fig. 3 SEM images of three samples. a: CA, b: PRF-acidizing-carb(1), and c: PRF-acidizing-carb(2). XRD patterns of carbon aerogels

XRD patterns of carbon aerogels are characterized by two broadened bands located at $23.5^{\circ}$ and $43.8^{\circ}$ (20) ascribable to the (002) and (101) reflections of graphite, respectively (Fig. 4). These wide peaks are commonly observed in disordered carbons[6]. The peak at $23.5^{\circ}(2 \theta)$ of PRF-acidizing-carb(2) is the sharpest, incidating the acidizing process of trifluoroacetic acid acetone solution can enhance the graphitization degree of carbon aerogels.

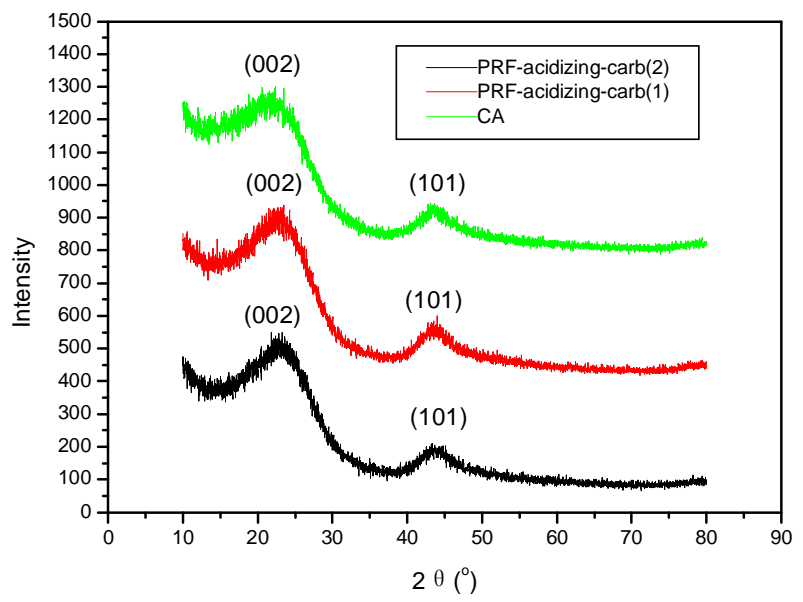

Fig. 4 XRD patterns of carbon aerogels

\section{Conclusions}

Carbon aerogel samples were prepared by the sol-gel polymerization method, resorcinol (R), phloroglucinol $(\mathrm{P})$, and formaldehyde $(\mathrm{F})$ were used as reactants. Acidizing process was introduced to the sol-gel reactions system.

The results show: (1) acidizing process of trifluoroacetic acid acetone solution decreased the shrinkage factor of carbon aerogel, increase absorption capacity and specific surface area; (2) the SEM images of carbon aerogel with acidizing process possess closer network structure and excellent connectivity, the holes between the network are abundant; (3) the XRD patterns show the acidizing process of trifluoroacetic acid acetone solution can enhance the graphitization degree of carbon aerogels.

\section{Acknowledgements}

This work was financially supported by Science-technology Support Plan Projects of Hebei Province (15291407D).

\section{References}

[1]R.W. Pekala, J. Mater. Sci. 24 (1989) 3221. 
[2]R.W. Pekala, Low density, resorcinol-formaldehyde aerogels, US patent 4,873,218, 1989.

[3]Frackowiak E, Beguin F. Carbon. 39 (2001) 937-950.

[4]K.S.W. Sing, D.H. Everett, R.A.W. Haul, L. Moscou, R.A. Pierotti, J. Rouquerol, T. Siemieniewska, Pure Appl. Chem. 57(1985) 603-619.

[5]Z.B. Zulamita, C.M. Francisco, M.C. Carlos. Materials Chemistry and Physics. 138 (2013) 870-876.

[6]F.J. Maldonado-Hódar, C. Moreno-Castilla, A.F. Perez-Cadenas. Microporous and Mesoporous Materials. 69 (2004) 119-125. 